\title{
Spatial and temporal structure of fish assemblages in a hyperhaline coastal system: Ría Lagartos, Mexico
}

\author{
Miguel Angel Peralta-Meixueiro and María Eugenia Vega-Cendejas
}

\begin{abstract}
The spatial and temporal fish species assemblages were analyzed throughout two annual cycles (2004-2005 and 2007-2008) in the Ría Lagartos Lagoon system, Mexico, via non-parametric multivariate analyses. We compared density and biomass of fish species among five habitat types defined by combinations of structure and environmental characteristics (hyperhaline, rocky, seagrass, channel, and marine), and three climatic seasons (dry, rainy, and northerlies). A total of 11,187 individuals distributed in 32 families and 63 species were collected. The most numerically abundant species were Floridichthys polyommus and Cyprinodon artifrons, while Sphoeroides testudineus contributed to the greatest biomass. Species composition consisted mainly of estuarine and euryhaline marine species. Spatially, a saline gradient was observed with marine conditions in the mouth, and increasing to over 100 in the inner zone of the system. Species richness, diversity and biomass declined from the mouth to the inner zone, while density showed an inverse tendency, with the highest values in the inner zone. Thus the salinity was the variable that best explained the spatial fish assemblages' structure. The ichthyofauna composition did not change over time, but the dominant species varied with the years. The abundance of juvenile specimens, suggest that the different habitats are used as feeding and breeding zones; hence it is proposed that protection strategies be pursued not only for the lagoon system but also for the northern zone of the Yucatan Peninsula.
\end{abstract}

Los ensamblajes espacio temporales de peces fueron analizados a través de dos ciclos anuales (2004-2005) y 2007-2008) en el sistema lagunar Ría Lagartos, México, vía análisis multivariados no paramétricos. Se comparó la densidad y biomasa de peces entre los cinco tipos de hábitats definidos por la combinación de características estructurales y ambientales (hiperhalino, rocoso, pastos, canal y marino) y tres temporadas climáticas (secas, lluvias y nortes). Un total de 11.187 individuos fueron recolectados, distribuidos en 32 familias y 63 especies. Las especies numéricamente más abundantes fueron Floridichthys poliommus y Cyprinodon artifrons, mientras que Sphoeroides testudineus contribuyó principalmente con la biomasa. La composición de especies consistió principalmente de especies marinas eurihalinas y estuarinas. Espacialmente, un gradiente de salinidad fue observado con condiciones marinas en la boca del sistema lagunar, incrementándose hacia la zona interna con valores superiores a 100. La riqueza de especies, diversidad y biomasa disminuyó desde la boca hacia la zona interna, mientras que la densidad mostró una tendencia inversa, con valores más altos en la zona interna. La salinidad fue la variable que mejor explicó la estructura espacial de los ensamblajes de peces. La composición de la ictiofauna no cambió en el tiempo, pero las especies dominantes varían con los años. La abundancia de especímenes juveniles sugiere que los diferentes hábitats son usados como zonas de alimentación y de crianza; por lo que se propone que la estrategia de protección continúe no sólo para el sistema lagunar sino para toda la zona norte de la Península de Yucatán.

Key words: Euryhaline ichthyofauna, Habitats, Seagrass, Yucatan Peninsula.

\section{Introduction}

The hyperhaline systems are complex and dynamic ecosystems (Simier et al., 2004). Their physical, chemical and biological properties present a strong gradient with variabilities that range from short periods of time to seasonal (Nogueira et al., 1997). They are located in arid and semiarid regions where the combination of high evaporation rates, poor oceanic circulation and a limited flow of freshwater results in salinities that frequently exceed 40 (Moyle \& Cech, 2000), and sometimes exceeds 100 (William, 1998; Valdés \& Real, 2004; Vega-Cendejas \& Hernández, 2004; Whitfield et al., 2006). Therefore, they have a great influence on the abundance, richness, and diversity, among other population and community parameters of the organisms, and can even become a determining factor for their presence or absence (Day et al., 1989; Ishitobi et al., 2000; Blaber, 2002; VegaCendejas \& Hernández, 2004; Potter et al., 2010).

Ichthyofauna has been considered as one of the principal biotic components of lagoon systems because it transfers

CINVESTAV-IPN, Unidad Mérida, Km. 6 Antig. Carr. A Progreso, A. P. 73 Cordemex, C. P. 97310 Mérida, Yucatán, México. mperalta@mda.cinvestav.mx 
nutrients to different levels of the food web, interchanges energy with neighbouring ecosystems and stores it within the ecosystem (Yáñez-Arancibia \& Nugent, 1977; Franco et al., 2006). It is recognised that a fish species can occupy one or several habitats during its life cycle (Blaber \& Blaber, 1980; Castro-Aguirre et al., 1999; Moyle \& Cech, 2000; Laegdsgaard \& Johnson, 2001; Cocheret de la Morinière et al., 2004). This is due to the fact that lagoon environments fulfil a wide range of functional roles in the life cycle of many species through the provision of shelter and/or conditions favourable for reproduction and spawning (Day \& Yáñez-Arancibia, 1985; Miller \& Skilleter, 2006).

The coastal Lagoon of Ría Lagartos is a hyperhaline system designated as a National Wildlife Refuge and protected by the Mexican Federal Government since 1979. Likewise, it is in the list of Wetlands of International importance and is a protected Special Biosphere Reserve where limited human activities are allowed (Frazier, 1999). It is characterized by high habitat heterogeneity with seagrasses, macrophytas and rocky cover contrasted with a hydrological variability from estuarine to hyperhaline conditions. The studies realized in the area, have described the ichthyofauna community and its association with environmental variables (Vega-Cendejas \& Hernández, 2004; García-Hernández et al., 2009). However, few studies have directly compared the structure of fish assemblages in different types habitats and contrasting for inter- annual periods.

This study aims to investigate the structure between the major habitat types of Ría Lagartos Lagoon (five) on a seasonal basis (dry, rainy, and northerlies). By sampling at different season of the year and comparing two annual periods, we examine the importance of the periodicity. These studies would provide much need information on the relative habitat value of seagrass and may reveal important spatial and temporal ecological connections between these prominent habitat types. Because of its wildlife refuge status and the ecologically importance of the seagrass beds as essential habitats for the ichthyofauna, this research is fundamental to establish management strategies of Ría Lagartos natural Reserve.

\section{Material and Methods}

\section{Study area}

Ría Lagartos is a coastal lagoon bordered by mangroves and seagrass bed zones (Halodule wrightii), approximately $80 \mathrm{~km}$ long by $25 \mathrm{~m}$ to $3.5 \mathrm{~km}$ wide (area: 9,467 ha) located along the north-eastern coast of the Yucatan Peninsula ( $21^{\circ} 26^{\prime}$ $-21^{\circ} 38^{\prime} \mathrm{NL}$ and $87^{\circ} 30^{\prime}-88^{\circ} 15^{\prime} \mathrm{WL}$ ) (Fig. 1). It is naturally connected to the sea via the mouth of San Felipe and artificially by a canal that remains open all year round in front of the town of Rio Lagartos (INE, 1994). It is characterised by the hyperhaline conditions recorded throughout much of its extension, which are the result of the geomorphology of the system, low precipitation, lack of rivers and high evaporation rate. The climatic regime is represented by three seasons: dry (March to June), rainy (July to October), and northerlies
(November to February) (Herrera \& Ramírez, 1997). The system presents a very marked salinity and substrate type gradient (Valdés \& Real, 2004). Hence, five types of habitat were identified based on salinity, transparency and substrate (Table 1): hyperhaline $(\mathrm{H})$, rocky $(\mathrm{R})$, seagrass $(\mathrm{S})$, channel $(\mathrm{C})$, and marine $(\mathrm{M})$.

\section{Sampling methods.}

The samples were attained bimonthly (2004-2005 and 20072008), with four times for each season, during the first $6 \mathrm{~h}$ of light using a beach seine ( $15 \times 2 \mathrm{~m}, 25 \mathrm{~mm}$ mesh size). Forty meters were trawled, covering an estimated area of $263 \mathrm{~m}^{2}$ with two replicas per station. Within the lagoon 10 stations were selected, two of which corresponded to each habitat type, as shown in Fig. 1. At each sampling station and prior to the collection of samples, water temperature $\left({ }^{\circ} \mathrm{C}\right)$, salinity and dissolved oxygen $\left(\mathrm{mg} \mathrm{l}^{-1}\right)$ were recorded mid-water with three replicas using a YSI multianalyzer (Yellow Spring Instrument) model 85.

All the fish caught were placed in labelled bags and fixed with $10 \%$ formalin. In the laboratory the samples were transferred to alcohol, identified to species level using specialized references (Fischer, 1978; Dickson \& Moore, 1998; McEachran \& Fechhelm, 2005), their standard length determined $(\mathrm{SL} \pm 0.1 \mathrm{~cm})$ and weighed $( \pm 0.1 \mathrm{~g})$. The fishes were deposited in scientific collection of Centro de Investigacion y de Estudios Avanzados del Instituto Politecnico Nacional (Clave: YUC. PEC 084 0999). The fish were divided into four ecological categories (Day et al., 1989) according to their origin and salinity tolerance: euryhaline, stenohaline, estuarine, and freshwater species.

Table 1. Structural and hydrological characteristics of the different habitats proposed in Ría Lagartos Lagoon, Mexico. *Refers the percentage cover in each habitat.

\begin{tabular}{lccl}
\hline Habitat & $\begin{array}{c}\text { Average } \\
\text { Salinity }\end{array}$ & $\begin{array}{c}\text { Transparency } \\
(\mathrm{cm})\end{array}$ & \multicolumn{1}{c}{ Substrate } \\
\hline Hyperhaline (H) & 90 & $<20$ & Sandy \\
Rocky (R) & 60 & $<40$ & Rocky bed \\
Seagrass (S) & 40 & $<70$ & Seagrass (90\%)* \\
Channel (C) & 35 & $>110$ & Muddy and seagrass (40\%)* \\
Marine (M) & 36 & $>110$ & Sandy \\
\hline
\end{tabular}

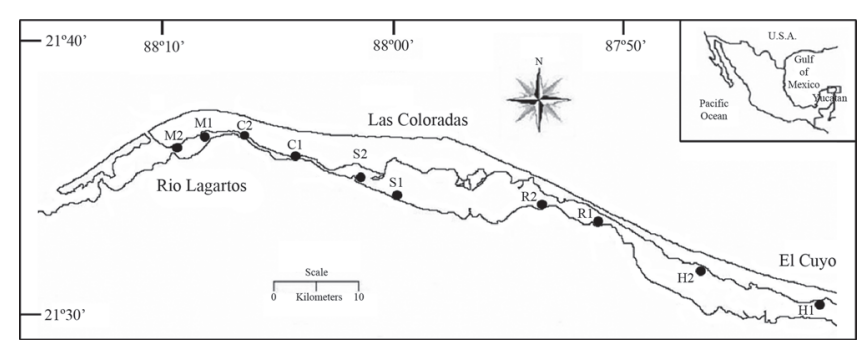

Fig. 1. Study area showing the Ría Lagartos Lagoon system, Mexico and sampling stations. Letters show different types of habitats and numbers are replicas in the same habitat. (hyperhaline: $\mathrm{H}$, rocky: $\mathrm{R}$, seagrass: $\mathrm{S}$, channel: $\mathrm{C}$, and marine: $\mathrm{M}$ ). 


\section{Data analysis}

To determine the spatial and temporal differences in the hydrographic variables, a one-way analysis of variance (ANOVA) was used, having previously verified the assumptions of normality (Kolmogorov Smirnov) and homogeneity of variance (Bartlett \& Cochran) (Zar, 1999). If these assumptions were not met, the data were transformed using $\log (\mathrm{x}+1)$. The Kruskall-Wallis test was used in cases where heterocedasticity was detected after transformation (Sokal \& Rohlf, 1998). The density and biomass data were standardized to $100 \mathrm{~m}^{2}$. The ecological parameters of the community, such as species richness $(S)$, Pielou's evenness index $(J)$ and the Shannon-Wiener index $\left(H^{\prime}\right)$ (Krebs, 1999), were analyzed temporally and spatially based on each habitat type. Dominance was estimated using the Importance Value Index (IVI), which takes into account the relative density (RD), biomass (RB), and frequency (RF) of each species (IVI $=\mathrm{RD}$ $+\mathrm{RB}+\mathrm{RF}$ ) (Brower \& Zar, 1977). This value ranges from 0 to 300 ; when divided by 3 this is referred to as the percentage of importance. The species that together formed at least $75 \%$ were considered as dominant.

Spatial and temporal variations in the structure of the fish assemblages were evaluated by non-metric multidimensional scaling (MDS) with 1000 iterations, derived from a Bray-Curtis similarity matrix constructed from the fish density data. Likewise, an analysis of similarity (ANOSIM) was performed between the fish of each habitat and climatic seasons (Clarke \& Green, 1988), with a non parametric test that uses a Bray-Curtis similarity matrix with density. This procedure consists of a statistical test $(\mathrm{R})$, which is analogous to an ANOVA. The null hypothesis tested was that no differences existed between the fish assemblages different zones (Quinn \& Keough, 2002). Species contribution by habitat and season was evaluated with a similarity percentage (SIMPER) test, which determines the percentage contribution of each species in order to classify a group (similarity) and discriminate species among sample groups (dissimilarity) (Clarke \& Green, 1988). These analyses were performed with the statistics program PRIMER v. 5 (Clarke \& Gorley, 2001).

Finally, to determine the relationship between fish species and environmental variables, a DCA was performed to estimate the length of the gradient. The resulting length was 4.3 , which indicates that the response is unimodal (Lepš \& Šmilauer, 2003); hence a Canonical Correspondence Analysis (CCA) was subsequently performed. The CCA was elaborated with the overall fish data matrix (dependent set) and the environmental data matrix (independent set). Interset correlations of this analysis were used to determine which environmental variables were the most important in determining the species abundance (ter Braak, 1995). The CANOCO software was used (ter Braak \& Smilauer, 1998) with the software options set for forward selection to test the significance of environmental variables.

\section{Results}

\section{Hydrological variables}

Differences in environmental variables were consistent between seasons $(P<0.001)$ (Table 2$)$. Temperature values were highest during the rainy season $\left(29.9^{\circ} \mathrm{C}\right)$ and lowest during the northerlies $\left(24.5^{\circ} \mathrm{C}\right)$. The highest average dissolved oxygen values were observed during the northerlies $(5.7 \mathrm{mg} / \mathrm{l})$, while the lowest were recorded during the dry season $(3.4 \mathrm{mg} / \mathrm{l})$ (Fig. 2). On the other hand, the highest average salinity values were found in dry season (59) and lowest in the rainy season (48). There were no significant spatial differences for temperature and dissolved oxygen.

Nevertheless, salinity presented a significant spatial gradient, with the highest values $(>87)$ recorded at the inner zone (rocky and hyperhaline habitat), which gradually decreased to marine values in the mouth of the lagoon (36). Significant spatial differences were found for diversity, evenness, density, and biomass but no temporal.

\section{Species composition and community descriptors}

A total of 11,187 specimens, distributed in 32 families, 51 genera, and 63 species, were collected (Table 3 ). The families with the greatest number of species were Gerreidae (8) and Sciaenidae (6), followed by Fundulidae, Cyprinodontidae, Syngnatidae, Carangidae, Sparidae, Paralichthyidae, and Tetraodontidae with 3 in each. The most numerically abundant

Table 2. Results of the ANOVA and Kruskal-Wallis* analyses to test for significant differences in environmental variables and for a number of ecological parameters of the fish community in Ría Lagartos Lagoon, Mexico.

\begin{tabular}{|c|c|c|}
\hline Variable/Analysis results & Spatial $(\mathrm{n}=5)$ & Temporal $(\mathrm{n}=3)$ \\
\hline \multicolumn{3}{|l|}{ Temperature } \\
\hline$F$ & 1.090 & 53.38 \\
\hline$p$ & 0.365 & $<0.001$ \\
\hline \multicolumn{3}{|l|}{ Dissolved oxygen } \\
\hline$H$ & $* 7.113$ & *15.433 \\
\hline$p$ & 0.129 & 0.001 \\
\hline \multicolumn{3}{|l|}{ Salinity } \\
\hline$H$ & *82.591 & $* 5.725$ \\
\hline$p$ & $<0.001$ & 0.571 \\
\hline \multicolumn{3}{|l|}{ Shannon Diversity $(H)$} \\
\hline$F$ & 24.06 & 0.010 \\
\hline$p$ & $<0.001$ & 0.986 \\
\hline \multicolumn{3}{|l|}{ Species Richness } \\
\hline$H-F$ & $* 29.267$ & 0.210 \\
\hline$P$ & $<0.001$ & 0.808 \\
\hline \multicolumn{3}{|l|}{ Eveness $(J)$} \\
\hline$H-F$ & $* 29.267$ & 0.990 \\
\hline$P$ & $<0.000$ & 0.378 \\
\hline \multicolumn{3}{|l|}{ Log Density } \\
\hline$F$ & 6.060 & 0.560 \\
\hline$P$ & 0.001 & 0.5737 \\
\hline \multicolumn{3}{|l|}{ Log Biomass } \\
\hline$F$ & 18.620 & 0.430 \\
\hline$P$ & $<0.000$ & 0.650 \\
\hline
\end{tabular}



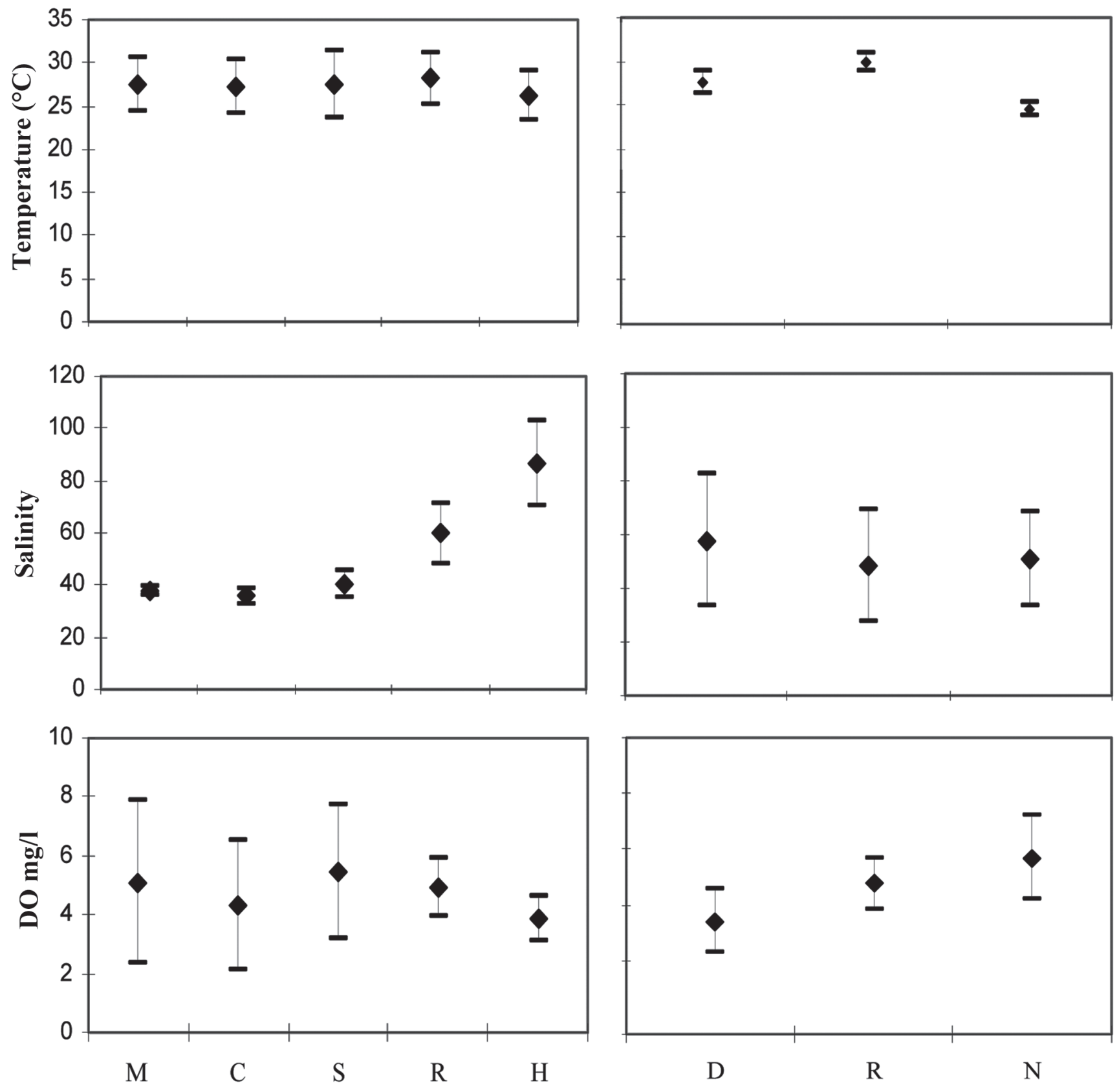

Fig. 2. Spatial (type of habitat) and seasonal variations in temperature, salinity, and dissolved oxygen ( \pm 1 STD).

species was Floridichthys polyommus (28.6\%), followed by Cyprinodon artifrons (26.9\%); the abundance of each of the remaining was less than $10 \%$. The total biomass caught was $137.7 \mathrm{~kg}$. Sphoeroides testudineus contributed the most biomass $(71.5 \%)$, followed by F. polyommus $(7.3 \%)$; the remaining species registered less than $3 \%$ each. The species with greater RF were $F$. polyommus $(12.4 \%)$, S. testudineus (10.3\%), and Eucinostomus gula (7.8\%).

According to the IVI, the dominant species were $S$. testudineus, F. polyommus, C. artifrons, E. gula, Eucinostomus argenteus, Eucinostomus harengulus, Achiurus lineatus, and Lagodon rhomboides. Altogether these species contributed more than $75 \%$ of the density. The community parameters such as diversity, richness and evenness, density and biomass only presented significant spatial differences (Table 2). Furthermore the lowest richness, diversity, evenness and biomass values were recorded in the hyperhaline habitat (Table 4), with a gradient that increased towards the mouth of the lagoon system. The density values were highest in the hyperhaline habitat due to the presence of a large number of juvenile $F$. polyommus and $C$. artifrons. In a seasonal base, the highest richness, diversity and evenness values were recorded, during the rainy season, while the lowest values were presented in the dry season. The greatest fish densities and biomasses were found 
Table 3. List of the fish species recorded in río Lagartos, Yucatan, ordered according to the classification of Nelson (2006). Size ranges are specified in standard length (SL), number of individuals (n), relative density (DR), relative biomass (BR) and relative frequency (FR), mean density (D: No. $100 \mathrm{~m}^{-2}$ ), life history stages (LS; J: juveniles; A: adults), ecological category code (EC; EH: Eurihaline; SH: Stenohaline; E: Estuarine; F: Freshwater) and habitat (H; M: marine; C: channel; S: seagrass; R: rocky; H: hyperhaline).

\begin{tabular}{|c|c|c|c|c|c|c|c|c|c|c|}
\hline Family & Species & SL (mm) & $\mathrm{n}$ & $\overline{\mathrm{DR}}$ & $\mathrm{BR}$ & FR & $\mathrm{D}$ & LS & EC & $\mathrm{H}$ \\
\hline Dasyatidae & Dasyatis americana Hildebrand \& Schroeder, 1928 & 650 & 2 & 0.02 & 2.22 & 0.29 & 0.01 & $\mathrm{~J}$ & EH & $\mathrm{M}, \mathrm{S}$ \\
\hline Gymnuride & Gymnura micrura (Bloch \& Schneider, 1801) & 155 & 1 & 0.01 & 0.90 & 0.15 & 0.01 & $\mathrm{~J}$ & SH & $\mathrm{M}$ \\
\hline Elopidae & Elops saurus Linnaeus, 1766 & $42-270$ & 29 & 0.26 & 0.38 & 2.33 & 0.19 & $\mathrm{~J}, \mathrm{~A}$ & EH & $\mathrm{M}, \mathrm{C}, \mathrm{S}, \mathrm{R}, \mathrm{H}$ \\
\hline Engraulidae & Anchoa mitchilli (Valenciennes, 1848) & $16-51$ & 78 & 0.70 & 0.01 & 1.16 & 0.51 & $\mathrm{~J}, \mathrm{~A}$ & EH & $\mathrm{M}, \mathrm{C}, \mathrm{S}, \mathrm{R}, \mathrm{H}$ \\
\hline Clupeidae & Harengula jaguana Poey, 1865 & $24-74$ & 12 & 0.11 & 0.06 & 0.44 & 0.08 & $\mathrm{~J}$ & EH & $\mathrm{M}, \mathrm{C}$ \\
\hline Ariidae & Ariopsis felis (Linnaeus, 1766) & $40-205$ & 101 & 0.90 & 1.44 & 1.31 & 0.66 & $\mathrm{~J}, \mathrm{~A}$ & EH & $\mathrm{C}, \mathrm{S}, \mathrm{R}$ \\
\hline Synodontidae & Synodus foetens (Linnaeus, 1766) & $34-253$ & 32 & 0.29 & 0.41 & 1.89 & 0.21 & $\mathrm{~J}, \mathrm{~A}$ & EH & $\mathrm{M}, \mathrm{C}, \mathrm{S}$ \\
\hline \multirow[t]{2}{*}{ Batrachoididae } & Opsanus beta (Goode \& Bean, 1880) & $25-200$ & 15 & 0.13 & 0.44 & 1.45 & 0.10 & $\mathrm{~J}, \mathrm{~A}$ & EH & $\mathrm{M}, \mathrm{C}, \mathrm{S}, \mathrm{R}$ \\
\hline & Opsanus phobetron Walters \& Robins, 1961 & 98 & 1 & 0.01 & 0.02 & 0.15 & 0.01 & $\mathrm{~J}$ & SH & M \\
\hline \multirow[t]{2}{*}{ Mugilidae } & Mugil curema Valenciennes, 1836 & $72-95$ & 4 & 0.04 & 0.03 & 0.29 & 0.03 & $\mathrm{~J}$ & EH & $\mathrm{M}, \mathrm{R}$ \\
\hline & Mugil trichodon Poey, 1875 & $67-192$ & 12 & 0.11 & 0.26 & 0.58 & 0.08 & $\mathrm{~J}, \mathrm{~A}$ & EH & $\mathrm{M}, \mathrm{C}$ \\
\hline \multirow[t]{2}{*}{ Atherinopsidae } & Menidia colei Hubbs, 1936 & $10-42$ & 259 & 2.32 & 0.05 & 4.07 & 1.70 & $\mathrm{~J}, \mathrm{~A}$ & EH & $\mathrm{M}, \mathrm{C}, \mathrm{S}, \mathrm{R}, \mathrm{H}$ \\
\hline & Menidia peninsulae (Goode \& Bean, 1879) & $20-33$ & 6 & 0.05 & 0.001 & 0.73 & 0.04 & $\mathrm{~J}, \mathrm{~A}$ & EH & $\mathrm{C}, \mathrm{R}, \mathrm{H}$ \\
\hline \multirow[t]{2}{*}{ Hemiramphidae } & Chriodorus atherinoides Goode \& Bean, 1882 & 116-187 & 11 & 0.10 & 0.17 & 0.44 & 0.07 & $\mathrm{~J}$ & EH & M \\
\hline & Hyporhamphus unifasciatus (Ranzani, 1841) & $180-242$ & 5 & 0.05 & 0.17 & 0.29 & 0.03 & $\mathrm{~J}$ & EH & $\mathrm{M}$ \\
\hline \multirow[t]{2}{*}{ Belonidae } & Strongylura notata (Poey, 1860) & $32-405$ & 63 & 0.56 & 1.68 & 3.34 & 0.42 & $\mathrm{~J}$ & EH & $\mathrm{M}, \mathrm{C}, \mathrm{S}, \mathrm{R}, \mathrm{H}$ \\
\hline & Strongylura tiтиси (Walbaum, 1792) & $300-355$ & 3 & 0.03 & 0.07 & 0.15 & 0.01 & $\mathrm{~J}$ & EH & $\mathrm{C}, \mathrm{S}$ \\
\hline \multirow[t]{3}{*}{ Fundulidae } & Fundulus grandissimus Hubbs, 1936 & $47-117$ & 25 & 0.22 & 0.20 & 1.02 & 0.16 & $\mathrm{~J}, \mathrm{~A}$ & E & $\mathrm{S}, \mathrm{R}, \mathrm{H}$ \\
\hline & Fundulus persimilis Miller, 1955 & $73-87$ & 3 & 0.03 & 0.02 & 0.29 & 0.02 & $\mathrm{~J}, \mathrm{~A}$ & $\mathrm{E}$ & M,S \\
\hline & Lucania parva (Baird \& Girard, 1855) & $11-58$ & 77 & 0.69 & 0.04 & 1.45 & 0.50 & $\mathrm{~J}, \mathrm{~A}$ & $\mathrm{E}$ & $\mathrm{M}, \mathrm{C}, \mathrm{S}, \mathrm{R}, \mathrm{H}$ \\
\hline \multirow[t]{3}{*}{ Cyprinodontidae } & Cyprinodon artifrons Hubbs, 1936 & $8-57$ & 3007 & 26.88 & 1.43 & 5.96 & 19.72 & $\mathrm{~J}, \mathrm{~A}$ & E & $\mathrm{C}, \mathrm{S}, \mathrm{R}, \mathrm{H}$ \\
\hline & Floridichthys polyommus Hubbs, 1936 & 7-91 & 3198 & 28.59 & 7.33 & 12.36 & 20.96 & $\mathrm{~J}, \mathrm{~A}$ & $\mathrm{E}$ & $\mathrm{M}, \mathrm{C}, \mathrm{S}, \mathrm{R}, \mathrm{H}$ \\
\hline & Garmanella pulchra Hubbs, 1936 & $6-30$ & 350 & 3.13 & 0.09 & 1.60 & 2.29 & $\mathrm{~J}, \mathrm{~A}$ & $\mathrm{E}$ & $\mathrm{M}, \mathrm{C}, \mathrm{S}$ \\
\hline Poeciliidae & Poecilia velifera (Regan, 1914) & $27-52$ & 58 & 0.52 & 0.05 & 1.02 & 0.38 & $\mathrm{~J}, \mathrm{~A}$ & $\mathrm{~F}$ & $\mathrm{M}, \mathrm{S}, \mathrm{R}$ \\
\hline \multirow[t]{3}{*}{ Syngnathidae } & Hipросатриs erectus Perry, 1810 & 82 & 1 & 0.01 & 0.01 & 0.15 & 0.01 & $\mathrm{~J}$ & SH & $\mathrm{C}$ \\
\hline & Syngnathus makaxi Herald \& Dawson, 1972 & $33-78$ & 26 & 0.23 & 0.003 & 1.16 & 0.17 & $\mathrm{~J}, \mathrm{~A}$ & EH & $\mathrm{M}, \mathrm{C}, \mathrm{S}, \mathrm{R}$ \\
\hline & Syngnathus scovelli (Evermann \& Kendall, 1896) & $45-62$ & 3 & 0.03 & $<0.001$ & 0.15 & 0.02 & $\mathrm{~J}, \mathrm{~A}$ & EH & $\mathrm{S}$ \\
\hline Triglidae & Prionotus tribulus Cuvier, 1829 & $15-85$ & 19 & 0.17 & 0.08 & 1.89 & 0.12 & $\mathrm{~J}$ & EH & M,C,S \\
\hline \multirow[t]{3}{*}{ Carangidae } & Caranx latus Agassiz, 1831 & 96 & 1 & 0.01 & 0.01 & 0.15 & 0.01 & $\mathrm{~J}$ & EH & $\mathrm{M}$ \\
\hline & Oligoplites saurus (Bloch \& Schneider, 1801) & 88 & 1 & 0.01 & 0.01 & 0.15 & 0.01 & $\mathrm{~J}$ & EH & $\mathrm{C}$ \\
\hline & Selene vomer (Linnaeus, 1758) & 23 & 1 & 0.01 & $<0.001$ & 0.15 & 0.01 & $\mathrm{~J}$ & EH & M \\
\hline Lutjanidae & Lutjanus griseus (Linnaeus, 1758) & $57-138$ & 10 & 0.09 & 0.19 & 0.73 & 0.06 & $\mathrm{~J}$ & EH & $\mathrm{M}, \mathrm{C}, \mathrm{S}$ \\
\hline \multirow[t]{8}{*}{ Gerreidae } & Diapterus auratus Ranzani, 1842 & $21-115$ & 63 & 0.56 & 0.20 & 2.04 & 0.41 & $\mathrm{~J}, \mathrm{~A}$ & EH & $\mathrm{C}, \mathrm{S}, \mathrm{R}, \mathrm{H}$ \\
\hline & Diapterus rhombeus (Cuvier, 1829) & $43-50$ & 4 & 0.06 & 0.01 & 0.29 & 0.03 & $\mathrm{~J}$ & EH & $\mathrm{R}$ \\
\hline & Eucinostomus argenteus Baird \& Girard, 1855 & $13-91$ & 542 & 4.85 & 1.25 & 6.25 & 3.55 & $\mathrm{~J}, \mathrm{~A}$ & EH & $\mathrm{M}, \mathrm{C}, \mathrm{S}, \mathrm{R}, \mathrm{H}$ \\
\hline & Eucinostomus gula (Quoy \& Gaimard, 1824) & $11-80$ & 789 & 7.05 & 2.35 & 7.85 & 5.17 & $\mathrm{~J}$ & EH & M,C,S,R \\
\hline & Eucinostomus harengulus Goode \& Bean, 1879 & $12-150$ & 455 & 4.07 & 1.46 & 5.38 & 2.98 & $\mathrm{~J}, \mathrm{~A}$ & EH & $\mathrm{M}, \mathrm{C}, \mathrm{S}, \mathrm{R}, \mathrm{H}$ \\
\hline & Eucinostomus melanopterus (Bleeker, 1863) & 69 & 1 & 0.01 & 0.005 & 0.15 & 0.01 & $\mathrm{~J}$ & EH & M \\
\hline & Eugerres plumieri (Cuvier, 1830) & $32-90$ & 20 & 0.18 & 0.10 & 1.31 & 0.13 & $\mathrm{~J}$ & EH & $\mathrm{C}, \mathrm{S}, \mathrm{R}$ \\
\hline & Gerres cinereus (Walbaum, 1792) & $53-100$ & 15 & 0.13 & 0.12 & 0.73 & 0.10 & $\mathrm{~J}$ & EH & $\mathrm{M}, \mathrm{C}$ \\
\hline Haemulidae & Orthopristis chrysoptera (Linnaeus, 1766) & $25-111$ & 12 & 0.11 & 0.08 & 0.44 & 0.08 & $\mathrm{~J}$ & EH & $\mathrm{M}, \mathrm{C}$ \\
\hline \multirow{3}{*}{ Sparidae } & Archosargus probatocephalus (Walbaum, 1792) & $48-85$ & 3 & 0.03 & 0.03 & 0.29 & 0.02 & $\mathrm{~J}$ & EH & $\mathrm{S}$ \\
\hline & Archosargus rhomboidalis (Linnaeus, 1758) & $44-78$ & 5 & 0.05 & 0.03 & 0.44 & 0.03 & $\mathrm{~J}$ & SH & $\mathrm{M}, \mathrm{S}$ \\
\hline & Lagodon rhomboides (Linnaeus, 1766) & $13-115$ & 398 & 3.56 & 2.26 & 3.34 & 2.61 & $\mathrm{~J}$ & EH & $\mathrm{M}, \mathrm{C}, \mathrm{S}$ \\
\hline Sciaenidae & Bairdiella chrysoura (Lacepède, 1802) & $50-101$ & 13 & 0.12 & 0.11 & 0.73 & 0.08 & $\mathrm{~J}$ & EH & $\mathrm{C}, \mathrm{S}$ \\
\hline & Bairdiella ronchus (Cuvier, 1830) & 133 & 1 & 0.01 & 0.04 & 0.15 & 0.01 & $\mathrm{~J}$ & EH & $\mathrm{S}$ \\
\hline & Cynoscion arenarius Ginsburg, 1930 & $31-99$ & 1 & 0.01 & 0.001 & 0.29 & 0.01 & $\mathrm{~J}$ & EH & $\mathrm{S}$ \\
\hline & Cynoscion nebulosus (Cuvier, 1830) & $24-248$ & 19 & 0.17 & 0.30 & 1.16 & 0.12 & $\mathrm{~J}, \mathrm{~A}$ & EH & $\mathrm{M}, \mathrm{S}, \mathrm{R}$ \\
\hline & Menticirrhus americanus (Linnaeus, 1758) & 30 & 1 & 0.01 & $<0.001$ & 0.15 & 0.01 & $\mathrm{~J}$ & EH & $\mathrm{C}$ \\
\hline & Micropogonias furnieri (Desmarest, 1823) & 23 & 1 & 0.01 & $<0.001$ & 0.15 & 0.01 & $\mathrm{~J}$ & EH & $\mathrm{S}$ \\
\hline Cichlidae & Cichlasoma urophthalmus (Günther, 1862) & $15-77$ & 17 & 0.15 & 0.68 & 0.58 & 0.11 & $\mathrm{~J}$ & $\mathrm{~F}$ & $\mathrm{C}, \mathrm{S}$ \\
\hline Gobiidae & Gobiosoma robustum Ginsburg, 1933 & $21-35$ & 12 & 0.11 & 0.003 & 0.73 & 0.08 & $\mathrm{~J}, \mathrm{~A}$ & EH & $\mathrm{M}, \mathrm{S}, \mathrm{R}$ \\
\hline Ephippidae & Chaetodipterus faber (Broussonet, 1782) & $50-277$ & 2 & 0.02 & 0.01 & 0.29 & 0.01 & $\mathrm{~J}$ & EH & $\mathrm{M}, \mathrm{S}$ \\
\hline Sphyraenidae & Sphyraena barracuda (Edwards, 1771) & $138-325$ & 10 & 0.09 & 0.65 & 1.31 & 0.07 & $\mathrm{~J}$ & SH & $\mathrm{M}, \mathrm{C}, \mathrm{R}$ \\
\hline Paralichthyidae & Citharichthys spilopterus Günther, 1862 & 142 & 1 & 0.01 & 0.03 & 0.15 & 0.01 & $\mathrm{~J}$ & EH & $\mathrm{S}$ \\
\hline & Paralichthys albigutta Jordan \& Gilbert, 1882 & $59-66$ & 4 & 0.04 & 0.01 & 0.29 & 0.03 & $\mathrm{~J}$ & EH & M \\
\hline & Syacium gunteri Ginsburg, 1933 & 130 & 1 & 0.01 & 0.03 & 0.15 & 0.59 & $\mathrm{~J}$ & EH & $\mathrm{C}$ \\
\hline Achiridae & Achirus lineatus (Linnaeus, 1758) & $15-93$ & 216 & 1.93 & 0.80 & 6.25 & 0.83 & $\mathrm{~J}$ & EH & $\mathrm{M}, \mathrm{C}, \mathrm{S}, \mathrm{R}$ \\
\hline Cynoglossidae & Symphurus plagiusa (Linnaeus, 1766) & $45-75$ & 5 & 0.05 & 0.01 & 0.58 & 0.03 & $\mathrm{~J}$ & EH & $\mathrm{M}, \mathrm{C}$ \\
\hline Tetraodontidae & Sphoeroides nephelus (Goode \& Bean, 1882) & $63-95$ & 2 & 0.02 & 0.04 & 0.15 & 0.01 & $\mathrm{~J}$ & SH & M \\
\hline & Sphoeroides spengleri (Bloch, 1785) & 67 & 1 & 0.01 & 0.02 & 0.15 & 0.01 & $\mathrm{~J}$ & EH & $\mathrm{R}$ \\
\hline & Sphoeroides testudineus (Linnaeus, 1758) & $25-213$ & 1149 & 10.27 & 71.48 & 10.32 & 7.53 & $\mathrm{~J}, \mathrm{~A}$ & EH & $\mathrm{M}, \mathrm{C}, \mathrm{S}, \mathrm{R}$ \\
\hline Diodontidae & Chilomycterus shoepfii (Walbaum, 1792) & $31-96$ & 9 & 0.08 & 0.19 & 0.73 & 0.06 & $\mathrm{~J}$ & EH & $\mathrm{M}, \mathrm{C}, \mathrm{S}$ \\
\hline & Total & & 11187 & 100 & 100 & 100 & & & & \\
\hline
\end{tabular}


in the dry season due to F. polyommus, E. gula, and $S$. testudineus juveniles that were the most abundant fish. We only recorded two freshwater species (Poecilia velifera and Cichlasoma urophtalmus), six were estuarine species, and the rest were marine species, the number of euryhaline species being the greatest.

\section{Spatial and temporal differences between assemblages}

The spatial analysis (MDS) based on species composition and abundance throughout the study period separated the different habitats with a stress value of 0.0 (Fig. 4a). Habitat H remained separated from the rest of the habitats. In the temporal MDS no separation was observed between the different sampling months that constituted the different seasons (Fig. 4b). The one-way ANOSIM with 999 permutations indicated significant differences in the composition of fish assemblages between the different habitats $(\mathrm{R}=432, P<0.03)$, except for seagrass - marine.

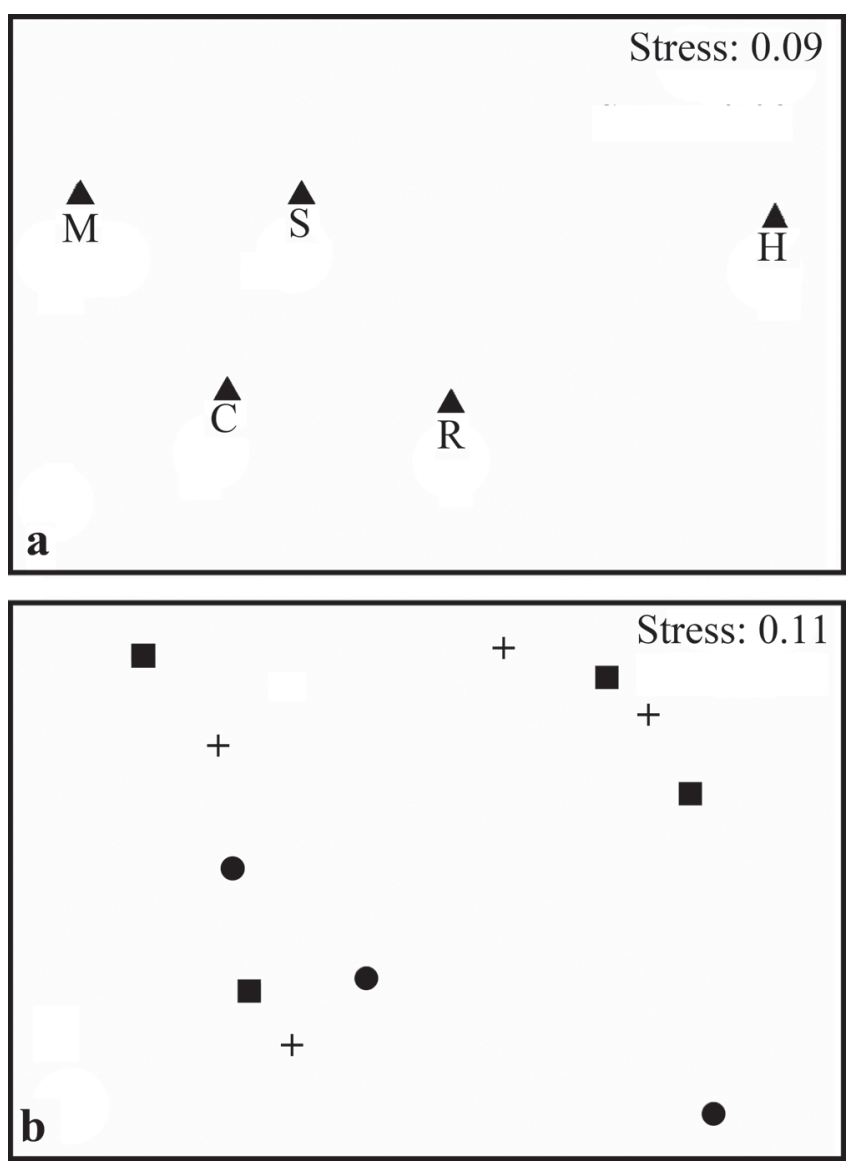

Fig. 3. Non-metric multi-dimensional scaling ordination plot derived from a Bray-Curtis similarity matrix constructed from the fish abundance data $(\log X+1$ transformation) in different types of habitat (a) and climatic seasons (b) in the Ría Lagartos system between October 2004 and December 2008. hyperhaline: $\mathrm{H}$, rocky: $\mathrm{R}$, seagrass: $\mathrm{S}$, channel: $\mathrm{C}$, marine: $\mathrm{M}$. $\%$ : Rainy, \% Northerlies, + Dry.
Table 4. Ecological parameters considering each type of habitat in the Ría Lagartos Lagoon, Mexico (hyperhaline: H, rocky: R, seagrass: S, channel: C, marine: $M$ ) and season (dry: $D$, rainy: R, northerlies: N).

\begin{tabular}{lcccccccc}
\hline & \multicolumn{4}{c}{ Habitat } & \multicolumn{3}{c}{ Season } \\
\cline { 2 - 9 } & $\mathrm{H}$ & $\mathrm{R}$ & $\mathrm{S}$ & $\mathrm{C}$ & $\mathrm{M}$ & $\mathrm{D}$ & $\mathrm{R}$ & $\mathrm{N}$ \\
\hline Species richness (N) & 13 & 26 & 40 & 37 & 42 & 44 & 50 & 45 \\
Diversity $(\mathrm{H})$ & 1.09 & 2.55 & 3.28 & 3.48 & 3.17 & 1.86 & 1.91 & 1.89 \\
Evenness & 0.29 & 0.54 & 0.62 & 0.67 & 0.59 & 0.55 & 0.64 & 0.58 \\
Density $\left(100 \mathrm{~m}^{2}\right)$ & 538.8 & 17.4 & 46.6 & 22.3 & 33.1 & 39.6 & 31.4 & 34.2 \\
Biomass $\left(100 \mathrm{~m}^{2}\right)$ & 60.9 & 186.1 & 605.9 & 221.5 & 1055.2 & 536.7 & 359.5 & 409.3 \\
\hline
\end{tabular}

Regarding the temporal analysis, no significant global differences were observed $(\mathrm{R}=0.02, P=0.12)$, however there were significant differences between the northerlies and dry season $(\mathrm{R}=0.068, P<0.02)$ (Table 5).

According to the SIMPER analysis, the total significant dissimilarity between the habitats ranged from 62.62 to $89.79 \%$; while between seasons this value was between 68.30 and 71.52. These differences are due to the abundance of dominant species in each habitat. The species that constituted more than $50 \%$ of the total spatial and temporal dissimilarity were $C$. artifrons, $F$. polyommus, S. testudineus, E. gula, E. argenteus, and $A$. lineatus. However, the species that characterised habitat $\mathrm{H}$ were $C$. artifrons and $F$. polyommus $(98 \%)$, while, F. polyommus constituted more than $52 \%$ of the dissimilarity in habitat R. For the rest of the habitats, no species had values above $36 \%$; in these habitats the dominant species were responsible for the principal contributions. The species that contributed most throughout the three seasons was $F$. polyommus, with contribution values greater than $40 \%$ during the rainy season. This was followed by $S$. testudineus with values that exceeded $21 \%$ during the northerlies. Similarly, $C$. artifrons and E. gula presented high values in all three seasons.

By performing the cluster analysis between habitats with the species abundance values, three groups were formed at $61 \%$ similarity (Fig. 5). The first included habitat $\mathrm{H}$, with a similarity of $40 \%$ with the rest of the habitats. Group II consisted of habitats $\mathrm{S}$ and $\mathrm{R}$, located in the centre of the system. Finally Group III consisted of habitats M and C, which are located to the west of the lagoon system with an important number of stenohaline marine species (occasional visitors).

\section{Relationship between species and hydrological variables}

According to the CCA, the first two axes explained 84.3\% of the accumulated variance in the species-environmental variables bi-plot (axis 1, 62.9\%: axis 2, 21.4\%). The correlation values showed salinity to be the most important environmental variable structuring the fish assemblages (axis 1, 0.861); in turn, temperature registered the highest value for axis $2(0.31)$. The direction of the two vectors (salinity and temperature) was almost perpendicular, indicating that the effects of these two factors on the fish assemblages are independent (Fig. 6). Both variables were significantly correlated with the ordination 
axes $(P<0.001)$. The composition of the ichthyofauna was inversely related to salinity, only the species $C$. artifrons and to a lesser extent $E$. saurus, showed preference for this variable. Florichthys polyommus, Diapterus auratus, and Menidia cole $i$ were strongly related to dissolved oxygen. The species Dasyatis americana, A. lineatus, and L. rhomboides were related to temperature. On a spatial basis, habitat $\mathrm{H}$ was highly correlated with salinity, habitats $\mathrm{M}$ and $\mathrm{C}$ were situated on the positive side of axis 1 , while habitats $\mathrm{R}$ and $\mathrm{S}$ were on the negative side. Temporally, the dry season was influenced by salinity, while the rainy and northerlies seasons were influenced by dissolved oxygen.

\section{Discussion}

In the Ría Lagartos Lagoon system the highest salinities are found in the inner zone and gradually decrease towards the mouth, creating a complex matrix of physiochemical factors that regulate the abundance and distribution of ichthyofauna assemblages. Although it is well known that underground rivers exist in the Yucatan Peninsula, their contribution to this ecosystem is very low; hence salinity had the greatest influence on species composition, as has been reported for other coastal lagoons by Paperno \& Brodie (2004), Simier et al. (2004), Vega-Cendejas \& Hernández (2004), and Whitfield

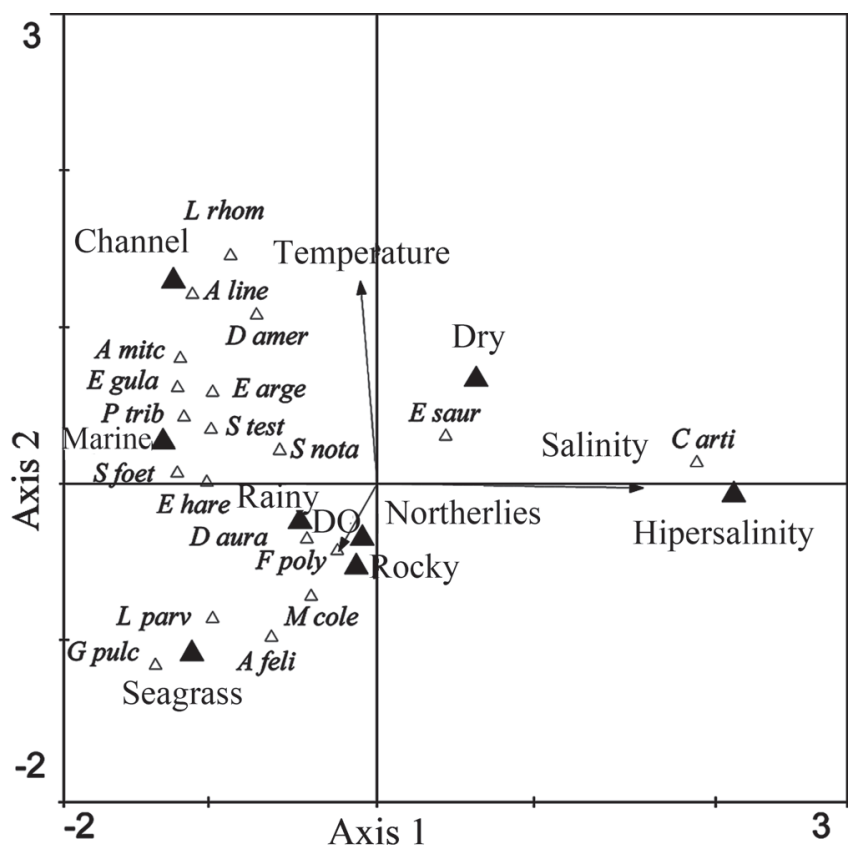

Fig. 4. Canonical Correspondence Analysis bi-plot. Length and direction of arrows indicate the relative importance and force of change in the environmental variables. CCA axis 1 and CCA axis 2 had eigen values of 0.629 and 0.214 respectively. The habitats and seasons are indicated. Only the species approximately $90 \%$ of IVI are shown. Species abbreviations are the first letter of the genus name and first four letters of the species name. DO: dissolved oxygen.
Table 5. R statistics values and their significance levels for pair-wise comparisons of species composition of the habitats and seasons using ANOSIM $(\mathrm{p}<0.05)$. hyperhaline: $\mathrm{H}$, rocky: $\mathrm{R}$, seagrass: S, channel: C, marine: M. ${ }^{*} \mathrm{p}<0.001,{ }^{*} \mathrm{p}<0.05$

\begin{tabular}{cc}
\hline Differences between habitats and between seasons & R value \\
\hline H - R & $0.312^{*}$ \\
H - S & $0.600^{*}$ \\
H - C & $0.895^{*}$ \\
H - M & $0.900^{*}$ \\
R - S & $0.115^{*}$ \\
R - C & $0.435^{*}$ \\
R - M & $0.393^{*}$ \\
S - C & $0.182^{*}$ \\
C - M & $0.236^{*}$ \\
Norths - Dry & $0.680^{* *}$ \\
\hline
\end{tabular}

et al. (2006). This was evident when a greater species richness was registered in the mouth zone (42 species), which decreased noticeably towards the interior of the lagoon (13 species). Similarly, fish diversity was lower in the hyperhaline zone (1.08) than in the marine zone (3.48), since marine species are distributed there (Franco et al., 2006).

The dominant species ( $F$. polyommus, $C$. artifrons, $S$. testudineus, E. gula, and E. argenteus) have also been reported by other authors as representative of these environments on a regional scale (Vega-Cendejas \& Hernández, 2004; Arceo-Carranza \& Vega-Cendejas, 2009). Both $F$. polyommus and $C$. artifrons are small estuarine species $(<10 \mathrm{~cm})$, but they have a high abundance $(>55 \%)$ due to their physiological capacity to tolerate high salinities and low dissolved oxygen values, hence they are abundantly distributed in hyperhaline and rocky habitats where the low species number reduces the competitive interactions (Colburn, 1988). Furthermore, it was observed that as the individuals of these species grew they moved to less saline habitats, probably in order to reduce competition with other cohorts or for food requirements.

García-Hernández et al. (2009), found that ichthyoplankton communities from four lagoon systems of Yucatan Peninsula, Ría Lagartos coastal system presented the highest density values, which suggests that it is important as a breeding and feeding zone for marine species, many of which are of commercial importance (Lutjanus griseus, Cynoscion arenarius, and C. nebulosus). In general the marine component (eurihaline and stenohaline) is distributed from the lagoon mouth to the seagrass habitat due to the steep increase in salinity towards the interior and the presence of the $H$. wrightii beds that offer them shelter and probably food. However, during the rainy season when salinity decreases to 42, marine species such as Ariopsis felis, Strongylura notata, and $S$. testudineus are recorded in the rocky habitat. As previously mentioned, freshwater seeps exist in habitats $\mathrm{C}$ and $\mathrm{M}$, and they reduced the salinity to almost 28. However because of the hyperhaline conditions, this influence is not so evident and only two eurihaline freshwater fish were recorded, unlike in other lagoon systems of the peninsula. 
The species richness obtained in this study is below average when compared to other studies carried out in the same geographic zone (Raz-Guzmán \& Huidobro, 2002; VegaCendejas, 2004; Arceo-Carranza \& Vega-Cendejas, 2009). This may be due to the zone sampled, fishing gear used, time of catch or the effort employed; however, much of the composition of fish in lagoon systems is due to the presence of occasional marine species that are only collected once during an entire sampling period (G. micrura, H. erectus, $C$. latus, $S$. vomer, C. arenarius, $M$. furnieri, among others), hence the size of the marine-lagoon connection and the time it is open for is a very important factor, since this allows lagoon-ocean communication (James et al., 2007; Mendoza et al., 2009). Simier et al. (2004) suggested that the high diversity recorded in lagoon systems may be due to: a) the establishment of marine species because of the permanent communication with the ocean and the presence of freshwater effluents; and b) the heterogeneity of habitats (seagrass beds, mangrove roots, muddy bottoms, and freshwater blooms) that favour the colonization of different species. However in Ría Lagartos Lagoon system the low richness is is due to high salinity throughout the year and throughout the lagoon system.

Gratwicke \& Speight (2005) confirmed habitat complexity, particularly the rugosity, variety of growth forms and height, to be the characteristics that favour species richness and diversity. The Ría Lagartos system has the afore mentioned characteristics; however, given that salinity rapidly increases towards the interior of the system due to its geomorphology and the high dominance of few species, species richness and diversity are not significant. Of the total species recorded, only eight are distributed throughout all of the habitats, while 21 species were collected in only one of the habitats.

The species composition shows no significant changes between years. However relative abundances showed strong inter-annual variations. This means that species that in previous years were not abundant, in later years are dominant. This is evident when compared with the study of VegaCendejas y Hernández (2004). This variation may be due to the presence of environmental changes caused by the hurricanes, such as opening a channel connection to sea which changes salinity gradient and allowing the movement of marine species to previously hyperhaline sites that shortly after the channel is closed by the settlers, the salinity gradually increases to the inner zoned. This again leads to a succession of species, but with a different structure.

The ANOSIM test shows significant variation between the habitats, which suggests that each habitat has hydrological and food characteristics that favour the development of the different species present in each of them. The seagrass and channel provided important habitats for fish specimens. In both of these habitats, because of the vegetation, a high diversity and higher densities were collected in the marine conditions of the lagoon. By contrast in the $\mathrm{R}$ habitat a low diversity and abundances were found (Table 4).

Similarly, the MDS analysis reflected a spatial separation of the fish assemblages. Although differences were observed between the fish assemblages on a seasonal basis (northerlies and dry), the spatial differences (habitats) were more significant, hence the structuring in this lagoon system is primarily spatial. As observed in the SIMPER results, although the fish assemblages are characterized by a group of dominant species, they are not what differentiate the different types of habitat, due to their broad distribution in the lagoon system; it is the rare species that differentiate the habitat types. For example, in habitat R, only Diapterus rhombeus and Sphoeroides spengleri were recorded; while in habitat S, Syngnathus scovelli, Archosargus probatocephalus, and Cynoscion arenarius were recorded, among others (Table 3). According to the CCA, salinity was the variable best related to species abundance (0.861), and as expected, the hyperhaline habitat presented a direct relationship with this variable. Although oxygen presented a weak relationship with fish abundance, it was strongly related to the northerlies season, due to the fact that the strong winds, which can last a week, increase the oxygen content of the water but decrease the temperature.

Management of a lagoon system requires the protection of the critical habitats of dependent species, including those that lie outside its physical boundaries. It also requires the control of both the quality and quantity of the water supplies on which its water balance and chemistry depend. Identification of these habitats and processes requires knowledge of the life histories of the organism and the hydrological regime. Understanding the function of each habitat and the relationships between them in a heterogeneous environment is essential for the integrated management of these ecosystems. Consequently, the conservation of each of these environments is fundamental for the survival and conservation of the ichthyofaunal diversity of the region.

\section{Acknowledgements}

We express our gratitude to Domingo Blanqueto, Elvia Mendoza, Daniel Arceo, Alex Acosta, and Gaspar Marfil for field assistance and laboratory support. We are also particularly grateful to Mirella Hernández de Santillana for her valuable help during the processing of the fish samples and to Consejo Nacional de Ciencia y Tecnología for the scholarship during the Doctoral Research of the first author. Similarly, we thank Gemma Franklin for her valuable help with the translation of the manuscript. Specimens were collected under legal authority of permits issued by the Secretaria de Pesca of Mexico.

\section{Literature Cited}

Arceo-Carranza, D. \& M. E. Vega-Cendejas. 2009. Spatial and temporal characterization of fish assemblages in a tropical coastal system influenced by freshwater inputs: northwestern Yucatan peninsula. Revista de Biología Tropical, 57: 89-103 
Blaber, S. J. \& T. J. Blaber. 1980. Factors affecting the distribution of juvenile estuarine and inshore fish. Journal of Fish Biology, 17: 43-162.

Blaber, S. J. 2002. Fish in hot water: the challenges facing fish and fisheries research in tropical estuaries. Journal of Fish Biology, 61: 1-20.

Brower, J. E. \& J. H. Zar. 1977. Field and Laboratory Methods for General Ecology. W. C. Brown Co. Publishers, Dubuque. Iowa. $320 \mathrm{p}$.

Castro-Aguirre, J. L., H. S. Espinosa-Pérez \& J. J. Shmitter-Soto. 1999. Ictiofauna Estuarino Lagunar y Vicaria de México. Colección Textos Politécnicos, Series Biológicas. LimusaNoriega. México, 711p.

Clarke, K. R. \& R. H. Green. 1988. Statistical Design and Analysis for a 'Biological Effects' Study. Marine Ecology Progress Series, 46: 280.

Clarke, K. R. \& R. Gorley. 2001. PRIMER V5: User Manual/ Tutorial. PRIMER-E. Plymouth, UK, 91p.

Cocheret de la Morinière, E., I. Nagelkerken, H. van der Meij \& H. van der Velde. 2004. What attracts juvenile coral ref. fish to mangroves: habitat complexity or shade? Marine Biology, 144: 139-145.

Colburn, E. A. 1988. Factors influencing species diversity in saline waters of Death Valley. Hydrobiologia, 158: 215-226.

Day, J. W. \& A. Yáñez-Arancibia. 1985. Coastal lagoons and estuaries as an environment for nekton. Pp. 17-34. In: Yáñez-Arancibia, A. (Ed.). Fish Community Ecology in Estuaries and Coastal Lagoons: Towards an Ecosystem Integration. Univ. Nal. Aut. Mex. Press. Mexico, 651p.

Day, J. W., C. A. Hall, W. M. Kemp \& A. Yáñez-Arancibia. 1989. Estuarine Ecology. Wiley-Interscience, New York, 558p.

Dickson, H. H. \& H. Moore. 1998. Fishes of the Gulf of México, Texas Louisiana and Adjacent Waters. Texas A \& M, University Press. USA, 422p.

Fischer, W. 1978. FAO Species Identification Sheets for Fishery Purposes. Western central Atlantic. FAO, Roma. Vol. IVII.

Franco, A., P. Franzoi, S. Malavasi, F. Riccato, P. Torricelli \& D. Mainardi. 2006. Use of shallow water habitats by fish assemblages in a Mediterranean coast lagoon. Estuarine Coastal and Shelf Science, 66: 67-83.

Frazier, S. 1999. A directory of wetlands of international importance: an update. Ramsar Convention Bureau. Compact disk.

García-Hernández, V., U. Ordóñez-López, T. Hernández-Vazquez \& J. Álvarez-Cadena. 2009. Fish larvae and juvenile checklist (Pisces) from the northern Yucatán Peninsula, Mexico, with 39 new records for the region. Revista Mexicana de Biodiversidad, 80: 85-94.

Gratwicke, B. \& M. R. Speight. 2005. The relationship between fish species richness, abundance and habitat complexity in a range of shallow tropical marine habitats. Journal of Fish Biology, 66: 650-667.

Herrera, S. J. \& J. R. Ramírez. 1997. Salinity and nutrients in the coastal lagoons of Yucatan, Mexico. Verhandelingen International Verein Limnology, 6: 1473-1478.

INE. 1994. Programa de Manejo de la Reserva Especial de la Biosfera de Ría Lagartos, Yucatán. Sedesol, Mexico, 140p.

Ishitobi, Y., J. Hiratsuka, H. Kuwabara \& M. Yamamuro. 2000. Comparison of fish fauna in three areas of adjacent eutrophic estuarine lagoons with different salinities. Journal of Marine Systems, 26: 171-181.
James, N. C., P. D. Cowley, A. K. Whitfield \& S. J. Lamberth. 2007. Fish communities in temporaly open/closed estuaries from warm-and cool-temperate regions of South Africa: a review. Review of Fish Biology and Fisheries, 17: 565-580.

Krebs, C. J. 1999. Ecological Methodology. Addison-Wesley, California, 620p.

Laegdsgaard, P. \& C. Johnson. 2001. Why do juvenile fish utilise mangrove habitats? Journal of Experimental in Marine Biology and Ecology, 257: 229-253.

Lepš, J. \& P. Šmilauer. 2003. Multivariate Analysis of Ecological Data using CANOCO. Cambridge University Press, UK, 395p.

McEachran, J. D. \& J. D. Fechhelm. 2005. Fishes of the Gulf of Mexico. University of Texas Press. Austin, 2116p.

Mendoza, E., M. Castillo-Rivera, R. Zárate-Hernández \& S. OrtizBurgos. 2009. Seasonal variations in the diversity, abundance, and composition of species in an estuarine fish community in the Tropical Eastern Pacific, Mexico. Ichthyology Research, 56: 330-339.

Miller, S. J. \& G. A. Skilleter. 2006. Temporal variation in habitat use by nekton in a subtropical estuarine system. Journal in Experimental Marine Biology and Ecology, 337: 82-95.

Moyle, P. B. \& J. J. Cech. 2000. Fishes: An Introduction to Ichthyology. Prentice Hall, Inc, USA, 512p.

Nogueira, E., F. Pérez \& A. Ríos. 1997. Seasonal patterns and long-term trends in an estuarine upwelling ecosystem (Ría de Vigo, NW Spain). Estuarine Coastal and Shelf Science, 38: 539-558.

Paperno, R. \& R. Brodie. 2004. Effects of environmental variables upon the spatial and temporal structure of a fish community in a small, freshwater tributary of the Indian River lagoon, Florida. Estuarine Coastal and Shelf Science, 61: 229-241.

Potter, I., B. Chuwen, S. Hoeksema \& M. Elliot. 2010. The concept of an estuary: A definition that incorporates systems which can become closes to the ocean and hypersaline. Estuarine Coastal and Shelf Science, 87: 497-500.

Quinn, G. \& M. Keough. 2002. Experimental Design and Data Analisis for Biologists. Cambridge University Press, UK, 527p.

Raz-Guzmán, A. \& L. Huidobro. 2002. Fish communities in two environmentally different estuarine systems of Mexico. Journal of Fish Biology, 61: 182-195.

Simier, M., L. Blanc, C. Aliaume, P. S. Diouf \& J. J. Albaret. 2004. Spatial and temporal structure of fish assemblages in an "inverse estuary", the Sine Saloum system (Senegal). Estuarine Coastal and Shelf Science, 59: 69-86.

Sokal, R. \& F. Rohlf. 1998. Biometry: The principles and practice of statistics in biological research, Third ed. Freeman and Company, New York, 745p.

ter Braak, C. J. F. 1995. Ordination. Pp. 71-173. In: Jongman, R., C. J. F. ter Braak \& O. Van Tongeren. (Eds.). Data Analysis in community and Landscape Ecology. Cambridge University Press London, 420p.

ter Braak, C. J. F. \& P. Smilauer. 1998. CANOCO reference manual and user's guide to Canoco for Winds: software for canonical community ordination, ver. 4. Microcomputer Power, Thaca, NY, $185 \mathrm{p}$.

Valdés, D. S. \& E. Real. 2004. Nitrogen and phosphorus in water and sediments at Ria Lagartos coastal lagoon, Yucatan, Gulf of Mexico. Indian Journal in Marine Science, 33: 338-345.

Vega-Cendejas, M. E. 2004. Ictiofauna de la Reserva de la Biosfera de Celestún, Yucatán: una contribución al conocimiento de su diversidad. Anales del Instituto de Biología, Universidad Nacional Autónoma de México, 75:193-206. 
Vega-Cendejas, M. E. \& M. Hernández. 2004. Fish community structure and dynamics in a coastal hypersaline lagoon: Rio Lagartos, Yucatán México. Estuarine Coastal and Shelf Science, 60: 285-299.

Williams, W. D. 1998. Salinity as a determinant of the structure of biological communities in salt lakes. Hydrobiologia, 381: 191201.

Whitfield, A. K., R. H. Taylor, C. Fox \& P. Cyrus. 2006. Fishes and salinities in the St Lucia estuarine system- a review. Review in Fish Biology and Fisheries, 16: 1-20.

Yáñez-Arancibia, A. \& R. S. Nugent. 1977. El papel ecológico de los peces en estuarios y lagunas costeras. Anales del Centro de Ciencias del Mar y Limnología, 4: 107-114.

Zar, J. H. 1999. Biostatistical analysis. Prentice-Hall, New Jersey, $620 \mathrm{p}$.

Submitted November 12, 2010

Accepted May 10, 2011

Published September 16, 2011 